\title{
Taking Time, Sharing Spaces: Adaptive Risk Governance Processes in Rural Japan
}

\author{
Ilan Chabay ${ }^{1}$
}

Published online: 9 November 2018

(C) The Author(s) 2018

\begin{abstract}
Rural and peri-urban communities in Japan, as well as in many other regions of the world, face risks of discrete event natural phenomena, including earthquakes, floods, and landslides. They also face persistent disruptive stress due to risks that remain active over long durations, such as the loss of community capacities due to an aging population. This article describes my observations of and subsequent reflections on adaptive risk governance and community resilience building processes in two areas of western and southern Japan-Chizu in Tottori Prefecture and towns near Kumamoto City in Kumamoto Prefecture. Four aspects of adaptive risk governance from this limited set of observations stood out: (1) the importance of establishing a durable, patient process, (2) initiated and facilitated by a trusted figure, in (3) a space or venue accessible and open to the community, and (4) augmented by boundary objects that facilitate role playing, iteration, and ownership by the community of solutions generated in these dialogues.
\end{abstract}

Keywords Boundary objects · Case Station-Field Campuses (CASiFiCA) · Community resilience $\cdot$ Knowledge, Learning, and Societal Change Alliance (KLASICA) · Japan · Risk governance $\cdot$ Yonmenkaigi System Method (YSM)

Ilan Chabay

ilan.chabay@iass-potsdam.de

1 Institute for Advanced Sustainability Studies (IASS), 14467 Potsdam, Germany

\section{Introduction}

Societies across the world are continually confronted by the threat of natural hazard-induced and anthropogenic disasters arising from a variety of sources and occurring over a range of temporal and spatial scales. How the risks of disastrous events are perceived and acted upon by different sectors of society in different contexts and cultures varies greatly. Some risks are related to changes on a global scale and manifest on that scale, yet even these global scale risks have significant local and regional impacts. In order to mitigate or adapt to risks, and to recover from and restore functionality after disasters, whether actual or potential events are global or local in origin, people and institutions at risk must engage in risk governance. This includes assessment, preparation, communication, response, recovery, and restoration appropriate to and effective in local conditions and culture. Accordingly, the process of governance of disaster risk and the decisions and actions taken by different institutions and individuals in communities at risk before, during, and after an event show considerable variation. Two areas in Japan provided contextualized examples for this article, which describes and frames an approach to adaptive risk governance in the face of persistent disruptive stressors in conjunction with the development of community resilience and the capability for such governance in a patient, inclusive, and incremental process.

This article is based on observations of two cases during a visit I made with Professor Norio Okada to two locations in western Japan in October 2017-one in the rural area around Chizu in Tottori Prefecture and the other on the semirural periphery of Kumamoto City in Kumamoto Prefecture (see Fig. 1). 


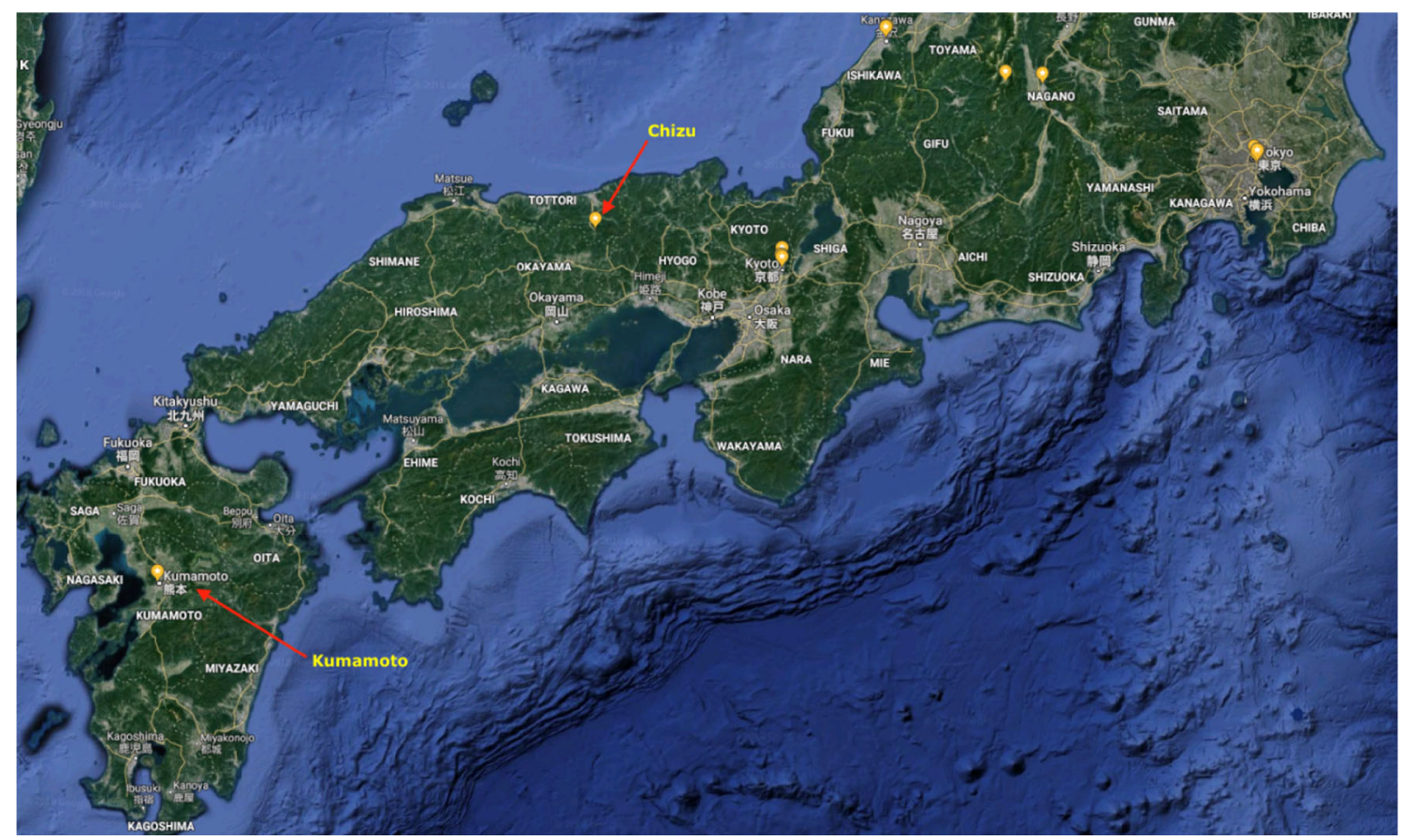

Fig. 1 Map of Japan with the locations of Chizu and Kumamoto noted (based on Google Maps)

Chizu is a location where Professor Okada and colleagues have worked for over 30 years with key individuals in the communities to develop innovative programs for adaptive risk governance. The Kumamoto area we visited was and is still in the process of recovery from a severe earthquake in 2016. Visiting these two places was a singular opportunity to observe the contexts and communities and to speak with people who had long been engaged in processes of dealing with both slowly-developing (for example, aging and declining community population) and rapid-onset (for example, earthquakes and landslides) threats. The short visits did not allow for in-depth case studies, but they did provide impressions of communitybased adaptive risk governance that raised questions and suggested issues for further exploration and analysis.

The methods developed by Professor Okada and colleagues and used in these locations have been described previously in several papers (for example, Xu et al. 2014; Higo et al. 2017), as well as having been employed in other locations (for example, Paton et al. 2010; Okada et al. 2013a; Samaddar et al. 2017). In this article, I wish to build on those foundational papers by reframing the approach and development of community capacity from the perspective of an "outsider" based on my limited observations. Two questions that I address in this article are: (1) what are the risks and long-term extrinsic stressors and consequent intrinsic stresses that pose critical challenges requiring adaptive risk governance in the two areas of Japan that I visited? and (2) what can be learned and reframed for more general consideration from the idiosyncratic contexts in which this adaptive governance approach and community resilience process developed?

\section{Observations}

My visits to several locations near Chizu and Kumamoto were opportunities to observe indications of the processes and progress in developing community resilience to the challenges of natural hazard risks and long-term trends. In this section, I describe the most significant observations. These observations were also reinforced by subsequent conversations with Japanese colleagues working on risk and resilience in this part of Japan.

\subsection{Chizu Area}

In this mountainous rural area, small villages are scattered in the valleys around the larger town of Chizu (population 7031 in $2016^{1}$ ) in Tottori Prefecture. Some of the villages consist of no more than three dozen houses separated from other villages by a few kilometers and mountainous topography. The people in this area have to contend with slow onset risks to the community from the shrinking numbers and aging of its population and rapid onset events, which include earthquakes, floods, and landslides. Over the period of three decades several steps have been taken and

\footnotetext{
${ }^{1}$ https://en.wikipedia.org/wiki/Chizu,_Tottori.
} 
become rooted in the communities that have established institutions and processes to address the threats. This has been a process that has greatly benefited from strong and able leadership from highly motivated individuals acting as essential local champions within the community inspired by and combined with creative, committed, and patient input from Professor Okada and others in academia. But crucially it has not been a process of top-down direction from community leaders or government, which had generally been people's experience and expectation in Japan. Rather it has been a long-term process of bringing together members of the community in dialogues, knowledge sharing, and self-governance with specific activities designed to facilitate fair and transparent decision making.

There are four aspects of the process of development and use of the process of adaptive governance that I observed and believe are particularly noteworthy. The first is that the process was intentionally patient and incremental development of a local "social movement," which was designed collaboratively by both the local champion, $\mathrm{Mr}$. Teretani, and Professor Okada. It is referred to as the "Zero-to-One" movement. The purpose was to take incremental steps starting from ground zero up in accord with the capacity and agency of the communities in the area at each point in time in order to begin to build confidence in the process and allow for a continual adaptation with changing circumstances.

The second aspect is the use of certain common spaces as venues for dialogues, building new enterprises in the community, and providing both tangible and intangible evidence of the ongoing viability of and hope for the future of the community. One former kindergarten building that was abandoned for lack of students has become the location for an innovative combination of café, brewery, and bakery using wild yeast harvested locally. Another abandoned secondary school has become repurposed as a hub for small enterprises, including an innovative eco-friendly restaurant and a pension business serving the community. And a forest kindergarten was created nearby, which draws children largely from the bigger city of Tottori (population 192,912 in $2016^{2}$ ), about $35 \mathrm{~km}$ from Chizu.

The third notable element is the maintenance of community identity and cohesion through rituals and celebrations at local shrines. The exact form of the celebration varies between villages, each in its own tradition, but they apparently serve to help each community to cohere by reconnecting with each other in the context of the community. They seem to remind people of their community identity and a narrative of their consistent, continuing place in a changing society (see Fig. 2).

\footnotetext{
${ }^{2}$ https://en.wikipedia.org/wiki/Tottori_(city).
}

And fourth, a valuable tool developed by a local champion and Professor Okada for adaptive risk governance, and called "Yonmenkaigi System Method" (YSM), was used to produce an open, transparent planning and adaptive governance process among stakeholders (Okada et al. 2013b). In this context, stakeholders refers to all those who are either influential in establishing policies and practices or who are potentially or actually affected by those policies and practices. YSM consists of a graphic boundary object in which the ideas and perspectives of the individuals are made evident for all present and recorded as a community creative design process for governance. It is used with a small group-four people at a time seated at a four-sided table. It is an iterative process by which the seated participants develop and record their ideas on a large sheet of paper through role playing. Each player takes on one of four functional roles in disaster risk governance or other community-relevant issues and records it in the closest quadrant of the paper. Iterations, including having the players exchange roles, are tracked in successive square rings (see Fig. 3 for an example). The purpose of playing is to facilitate the dialogue among stakeholders and to structure planning for adaptive governance, including disaster risk governance, as well as for other community challenges. Preserving the recording of input and progress on the paper in single or multiple workshops, as well as signing the recording and the photographs of the group, serves to provide not only systematic documentation, but also reinforces commitments to and ownership of the outcomes.

\subsection{Kumamoto Area}

We visited several towns and temples east and northeast of Kumamoto City, as well as Kumamoto University. As of 1 April 2017, Kumamoto City has an estimated population of 737,812. Greater Kumamoto had a population of 1,461,000 in the 2000 census. $^{3}$ A series of earthquakes struck the area beginning 14 April 2016, including a tremor with moment magnitude 7.0 early in 16 April $2016 .^{4}$ Extensive damage was done across a wide area to several large and small temples, the main building of the large Kumamoto castle, and many homes, apartment buildings, and businesses.

An initiative called Case Station-Field Campus (CASiFiCA) played an important role in the response, recovery, and restoration following the earthquake. CASiFiCA was first established by Professor Okada at Kumamoto University through a Japanese government ministry grant in 2013, after he moved to Kumamoto University from the Disaster Prevention Research Institute (DPRI) at Kyoto

\footnotetext{
3 https://en.wikipedia.org/wiki/Kumamoto.

${ }^{4}$ https://en.wikipedia.org/wiki/2016_Kumamoto_earthquakes.
} 
Fig. 2 Ceremony in Itaibara village, near Chizu. Photograph by the author, October 2017
Fig. 3 Yonmenkaigi record from Yamasato, near Chizu, showing the quadrant and ring structure. Photograph by the author, October 2017
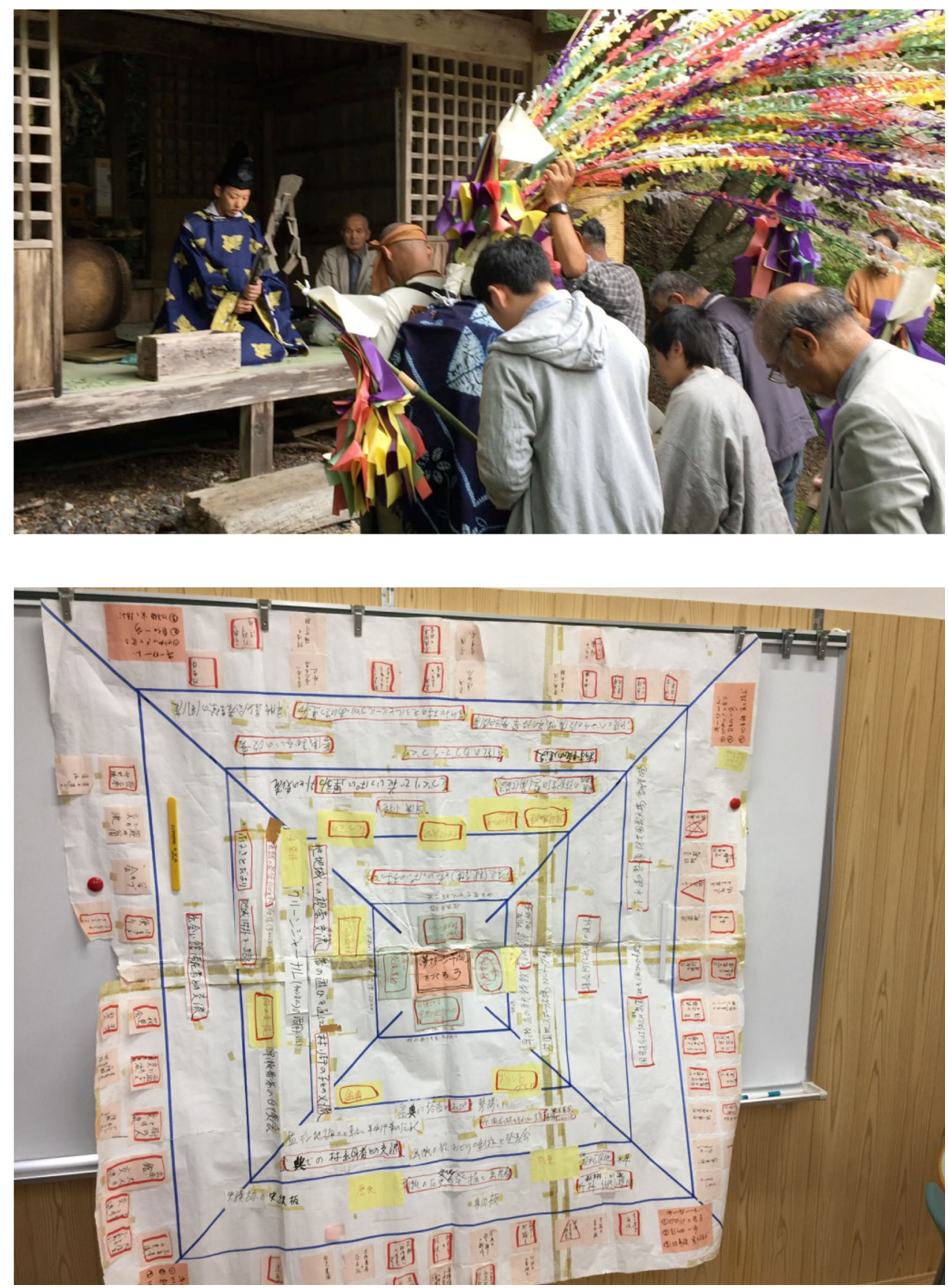

University. The acronym CASiFiCA compactly denotes the function of the program, that is, it pairs local cases, such as the growth of the Zero-to-One movement, with academic scientists to encourage and facilitate on-going empirical studies and analysis of the societal changes in the local communities.

Fortunately, the CASiFiCA program became an educational resource that facilitated rapid self-organization among students and professors for rescue and relief for victims of the 2016 earthquake. Subsequently a Case Station-Field Campus was also organized in Chizu. Additional links to other universities in Japan and beyond are being developed.

Two observations about the aftermath of this earthquake stand out. One is the importance of building a broad-based community process of emotional recovery, as well as mapping the physical damage and compiling community needs. A young Buddhist monk, living in a makeshift shelter, used the shelter as a base for his efforts to record the damage and community needs, plan reconstruction, and build a long-term process of recovery well after the crisis. He became an important central resource for the 
Fig. 4 Professor Okada and Buddhist monk examining topographical map of damage locations in community meeting space. Photograph by the author, October 2017

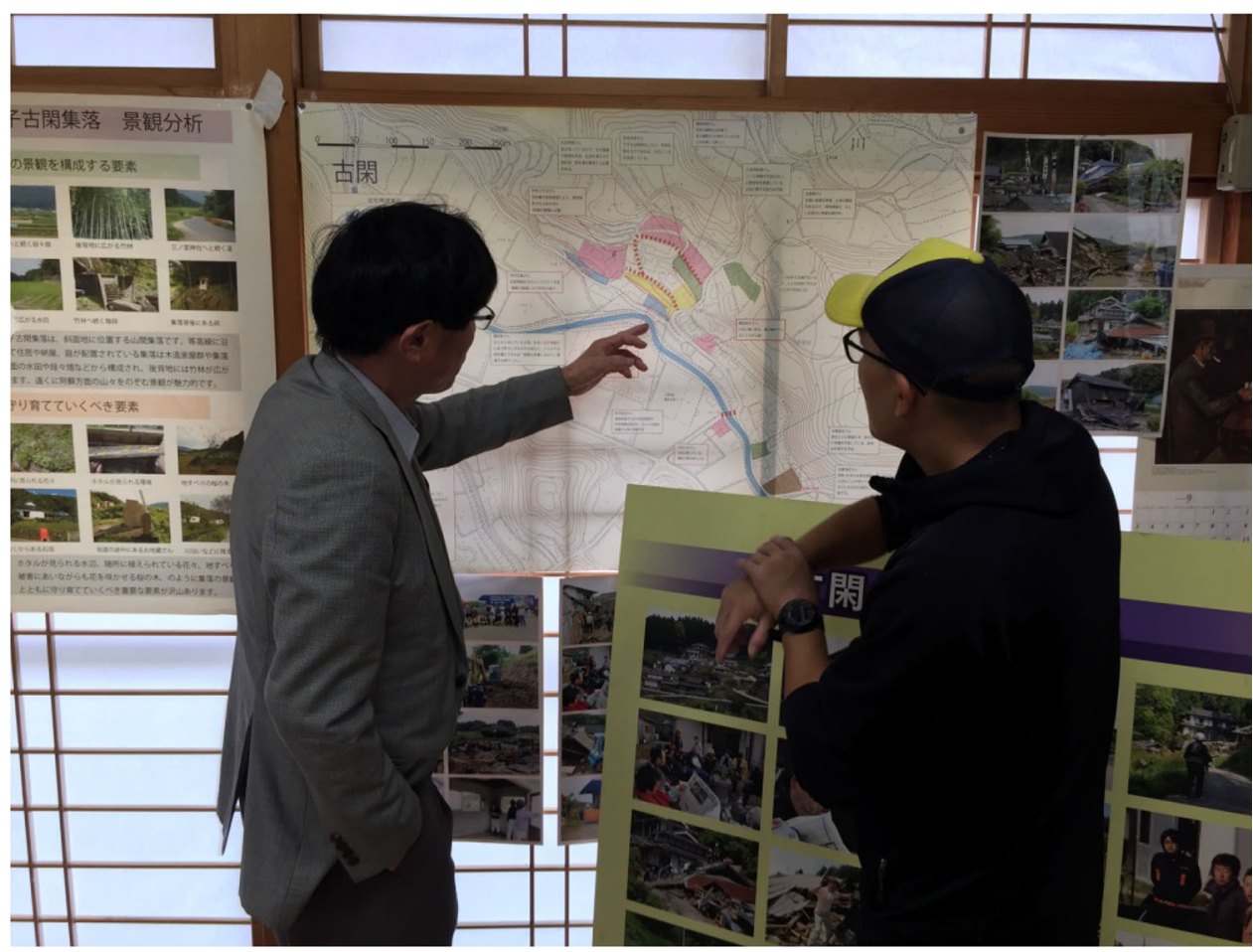

communities dealing with recovery and rebuilding of the physical infrastructure, health, and emotional aspects. Photographs and maps covered the walls and documented the community events and progress over the year and a half following the earthquake. The monk used the shelter as a space in which he could attend to the community's social, emotional, and spiritual needs and facilitate a creative social process that was leading over time to the design for recovery and rebuilding of the community to a condition improved upon compared to its pre-earthquake status (Fig. 4).

A second observation concerned the people whose living spaces, whether houses or apartments, became uninhabitable. One positive aspect was the rapid construction of temporary housing in converted shipping crates with essential services, commercial facilities, community center (Fig. 5), and bus service to other locations in the Kumamoto area. More problematic, particularly for the elderly, were the long times-more than one year for some-needed to find new permanent housing and, in some cases, people's reluctance and delay in accepting and acting on the need to start anew in an unfamiliar living space.

\section{Reflections}

Most of the people engaged in formal and informal meetings and dialogues have been part of their community for a number of years and consequently have built up a set of relationships with others in the community. As a consequence of this interpersonal social history, and of the cultural norms in which shared responsibility and communication among those in the community is highly valued and widely practiced, substantial social capital is available in the lives of communities in both rural areas and urban neighborhoods.

Social capital and long-standing relationships seem to enable dialogues that are open and offer opportunities to express a range of values. This is even more the case when the dialogues are facilitated by use of a boundary object, such as the Yonmenkaigi System Method. It is crucial that these dialogues and communications are a means of continually learning and refining participatory governance, not only exchanging often decontextualized information on prescribed responses to risk. This provides a means of generating ownership of adaptive responses appropriate to the local conditions and links several levels of government and institutions in the local process.

The repurposing (for example, abandoned schools near Chizu) and low-cost constructions (for example, shipping containers in Mashiki, near Kumamoto, see Fig. 6) of community spaces have become important venues that now serve as common space for gathering and communication among members of the community. They have also become places of remembrance for those impacted by disaster and a site for gathering to recover and rebuild.

The Case Station-Field Campuses are institutions that are transdisciplinary in nature and thereby serve as bridges 
Fig. 5 New community center in a temporary housing town near Kumamoto airport built for people displaced by the earthquake. Photograph by the author, October 2017

Fig. 6 Shipping containers serving as a community center in Mashiki town, near

Kumamoto. Photograph by the author, October 2017
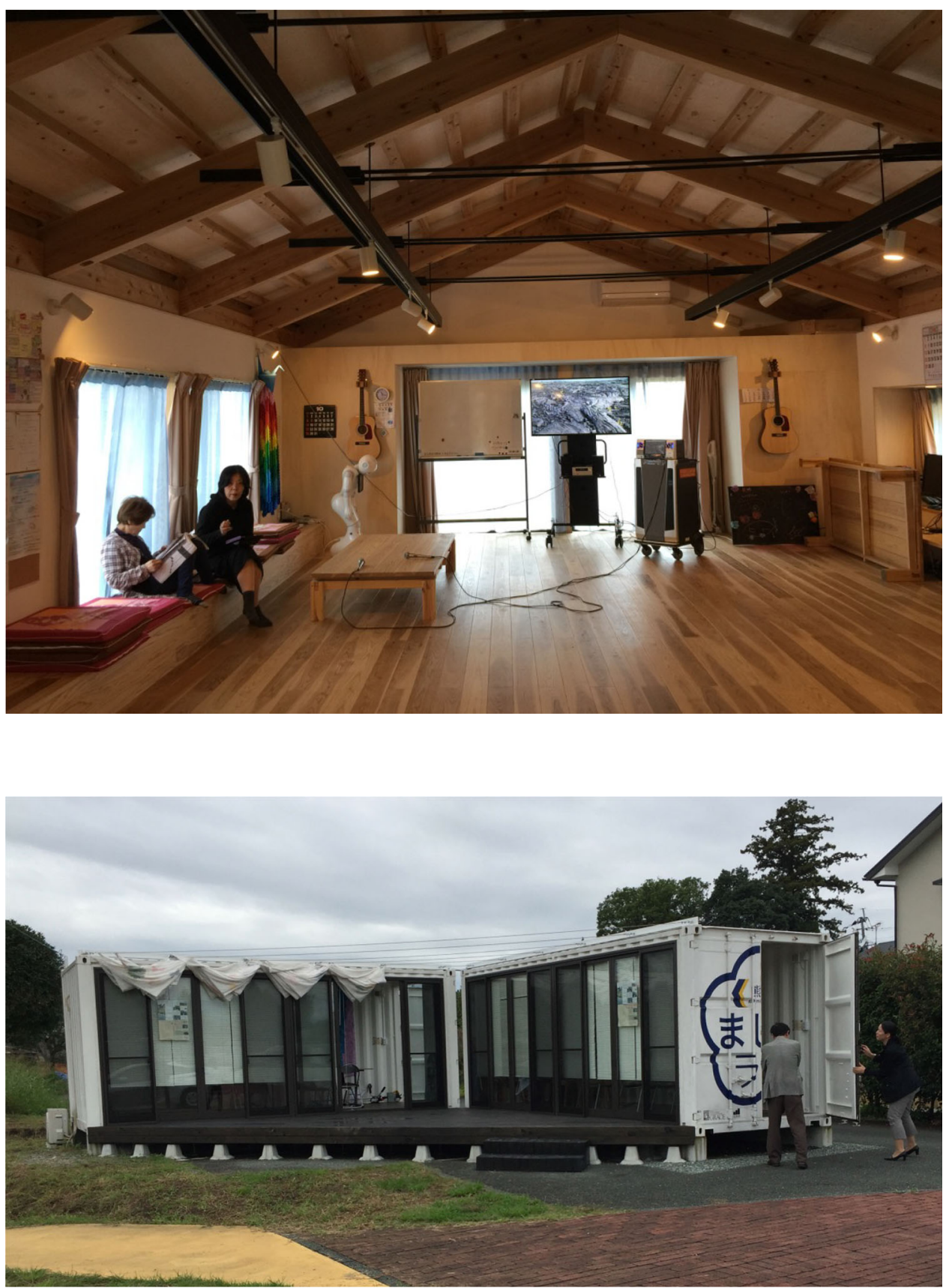

between local community members, governmental institutions, and academics in multiple intellectual domains. The links between multiple case locations, case station field campuses, and universities lead to several important benefits. It allows for comparison of cases, growth of interdisciplinary connections, and curriculum development between natural sciences, engineering, social sciences, health sciences, and social welfare programs, primarily focused on disaster risk governance in a broad perspective.
CASiFiCA and the Knowledge, Learning, and Societal Change Alliance (KLASICA), ${ }^{5}$ a project directed by the author and based in the Institute for Advanced Sustainability Studies (IASS) in Potsdam, Germany, share a core concern with understanding and facilitating social movements or collective behavior change in communities.

\footnotetext{
5 https://www.iass-potsdam.de/en/research/knowledge-learning-andsocietal-change-alliance-klasica.
} 
CASiFiCA is centered in adaptive disaster risk governance, while KLASICA is concerned with understanding and facilitating collective behavior transitions toward sustainable futures in local and regional contexts and cultures. They share inter- and transdisciplinary approaches and most importantly, both emphasize transformative processes of meaning making and decision making in a participatory, adaptive governance process for long-term strategic benefit of the community. Many examples of such transformative processes arise in a wide range of locations and conditions and are described and framed in the context of the KLASICA project and the Integrated Local and Environmental Knowledge (ILEK) project in a new book by Sato et al. (2018).

Another area of common purpose, although manifested in different forms, is the use of boundary objects-2- or 3-dimensional representations with which dialogue can be facilitated-with iterative play to stimulate and structure dialogue. As described above, YSM is such an iterative, structured, multiplayer format used to address adaptive governance. The author has also developed several different group game formats for the purpose of stimulating dialogue among different stakeholder groups and using the interactions to understand and facilitate change processes.

One example is a mobile exhibition in Baden-Württemberg, Germany designed by the author and Professor Ortwin Renn, which includes an energy transition (Energiewende) game. The energy transition game (Li et al. 2015) combines electronic displays, which present the player with household tasks to perform that require energy in the $5 \mathrm{~min}$ game (representing a virtual week). The unique feature with consequences that stimulate questions from the players is that the combined power demand of all the player households is supplied by a mix of renewable and coal-fired power plants represented by a hand-cranked generator operated by one of the players. If there is no coordination of energy use, the result is that the person supplying the power is unable to meet the demand and a "blackout" occurs, intentionally leading to discussion of how to change strategies to avoid such high peak demands.

Another example developed by the author with Professor Vittorio Loreto and colleagues in the Kreyon project ${ }^{6}$ at the University of Rome is a game that uses landscapes constructed with LEGO blocks and an augmented reality system to illustrate in a simple manner the consequencesoften unintended or unanticipated-of making decisions and taking actions in complex systems. The augmented reality consists of a video camera (analogous to a satellite system) that projects information in a graphical display indicating total $\mathrm{CO}_{2}$ output of the landscape, water flow

\footnotetext{
${ }^{6}$ http://www.kreyon.net/en/kreyon/kreyon-project.
}

and water quality (if there is a river in the landscape), energy demand, air pollution, and so on. ${ }^{7}$

\section{Conclusion}

Four ideas stand out for me from my experiences in Chizu and Kumamoto.

1. The importance of establishing and sharing common spaces or venues for creative social dialogues. These are spaces in which all members of the community can feel welcome and comfortable;

2. The willingness of members of the community to take the time and invest the patience to allow the community to undergo an iterative, on-going process that builds capacity for attending to a variety of community issues, including regrouping, redesigning, and rebuilding together in the aftermath of disasters or devising measures to reduce sources of long-term stress;

3. The importance of local champions and trusted facilitator(s) from within or beyond the community; and

4. The important benefits that certain boundary objects provide is to make issues and concepts more concrete, accessible, and visible to all present, thus helping to catalyze more constructive dialogues. The boundary object can be a symbol or model that embodies concepts and information in a form that stimulates creative thinking and testing of ideas by the members of the community affected by or influential in the conditions being addressed.

The ideas listed above are based in the recognition of the importance of human-centered, socially aware decision making in a field of multiple possible solutions guided by the relevant community and its values. This is in contrast to relying only on externally directed technocratic solutions. They illustrate the value placed on a process of incremental, anticipatory, and adaptive governance in addressing the multiple risks facing the communities of Chizu and Kumamoto.

Open Access This article is distributed under the terms of the Creative Commons Attribution 4.0 International License (http://crea tivecommons.org/licenses/by/4.0/), which permits unrestricted use, distribution, and reproduction in any medium, provided you give appropriate credit to the original author(s) and the source, provide a link to the Creative Commons license, and indicate if changes were made.

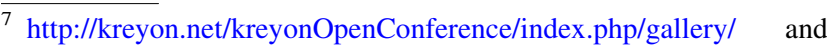
http://nova.ilsole24ore.com/progetti/lo-sviluppo-parte-dai-contesti/ ?refresh_ce=1.
} 


\section{References}

Higo, E., N. Okada, K.W. Hipel, and L.P. Fang. 2017. Cooperative survival principles for underground flooding: Vitae system based multi-agent simulation. Expert Systems with Applications 83: 379-395.

Li, H., I. Chabay, O. Renn, A. Weber, and G. Mbungu. 2015. Exploring smart grids with simulations in a mobile science exhibition. Energy, Sustainability and Society 5: 37. https://doi. org/10.1186/s13705-015-0066-4.

Okada, N., L.P. Fang, and D.M. Kilgour. 2013a. Community-based decision making in Japan. Journal of Decision and Negotiation 22(1): 45-52.

Okada, N., J.-L. Na, and L. Fang, and A. Teratani. 2013b. The Yonmenkaigi system method: An implementation-oriented group decision support approach. Journal of Decision and Negotiation 22(1): 53-67.
Paton, D., R. Bajek, N. Okada, and D. McIvor. 2010. Predicting community earthquake preparedness: A cross-cultural comparison of Japan and New Zealand. Natural Hazards 54(3): 765-781.

Samaddar, S, N. Okada, J. Choi, and H. Tatano. 2017. What constitutes successful participatory disaster risk management? Insights from post-earthquake reconstruction work in rural Gujarat, India. Natural Hazards 85(1): 111-138.

Sato, T., I. Chabay, and J. Helgeson (eds.). 2018. Transformations of social-ecological systems: Studies in co-creating integrated knowledge toward sustainable futures. In Ecological research monographs. Singapore: Springer.

Xu, W., Y. Li, N. Okada, Y. Takeuchi, Y. Kajitani, and P.J. Shi. 2014. Collaborative modelling-based shelter planning analysis: A case study of the Nagata Elementary School community in Kobe City, Japan. Disasters 38(1): 125-147. 